\title{
Parameter Estimation of Maneuvering Target Using Maximum Likelihood Estimation for MIMO Radar with Colocated Antennas
}

\author{
Hai Li, Ling Xu, Zhe Zhang \\ Tianjin Key Lab. for Advanced Signal Processing, Civil Aviation University of China, Tianjin, China \\ Email: elisha1976@163.com
}

How to cite this paper: Li, H., Xu, L. and Zhang, Z. (2017) Parameter Estimation of Maneuvering Target Using Maximum Likelihood Estimation for MIMO Radar with Colocated Antennas. Journal of Computer and Communications, 5, 69-74. https://doi.org/10.4236/jcc.2017.53008

Received: March 2, 2017

Accepted: March 10, 2017

Published: March 13, 2017

\begin{abstract}
The estimation of target parameters in MIMO radar signal processing is one of the most important research topics. An efficient implementation of the Maximum Likelihood estimator is presented in this paper to estimate the DOA (Direction of Arrival), initial velocity and acceleration of a maneuvering target in colocated MIMO radar. The target's DOA is estimated in the first place, then a Maximum-Likelihood (ML) estimation based on peak search is applied to a two-dimensional grids providing estimation of initial velocity and acceleration. Simulations show that the MIMO radar has a better performance in DOA estimation than the phased array radar. By means of Monte Carlo simulations, the estimation error of initial velocity and acceleration on different SNRs are calculated. The results also suggest the effectiveness of this method.
\end{abstract}

\section{Keywords}

MIMO Radar, Parameter Estimation, Maximum Likelihood Estimation

\section{Introduction}

The Multiple-Input Multiple-Output (MIMO) Radio Transmission Technology, has the potential to enhance system capacity and improve spectral efficiency without requiring extra emission signal bandwidth or transmission power [1] [2]. The overwhelming popularity of MIMO systems draws radar researchers' attention to engage MIMO techniques in radar systems implementation.

MIMO radar employs multiple elements at both transmitting and receiving antennas as the phased array radar does, on flip side, MIMO radar transmits orthogonal or partially correlated waveforms. According to the antenna configuration, MIMO radar systems can be classified into two categories: collocated (co- 
herent) MIMO radars are those with closely spaced antennas [3]. While distributed MIMO radars, also known as non-coherent MIMO radars, whose antennas are placed far from each other [4]. The latter configuration exploits the random fluctuation of the targets' Radar Cross Section (RCS). This fluctuation causes spatial decorrelation between target echoes, which prevents coherent processing. It is known that spatial resolution can be improved by taking advantage of coherent processing introduced by coherent MIMO radar. Several methods have been proposed for DOA estimation offering significant estimate performance without considering Doppler effects [5] [6]. As shown in [7] and [8], parameters of moving target can be measured by the maximum likelihood estimator for distributed MIMO radar. There are many efforts have been made on joint DOA and Doppler frequency estimation [9] [10], while few studies have yet been conducted on maneuvering targets.

This paper considers parameters estimation of maneuvering target in colocated MIMO radar by developing a maximum-likelihood estimator. The MUltiple SIgnal Classification (MUSIC) is applied in the first place to estimate DOA, followed by a two-dimensional search applied to estimate the velocity and acceleration. Performance of estimation for phased array radar and MIMO radar are compared under the same condition. The root mean square errors (RMSE) of the ML estimation of target parameters are obtained by Monte Carlo simulations. Numerical results demonstrate that MIMO radar has better angle estimation performance compared with conventional phased array radar and verify the effectiveness of this method as well.

\section{MIMO Radar Signal Model}

Let's consider a MIMO radar system in which there are $M$ transmitting antennas and $N$ receiving antennas, with inter-element spacing of $d_{t}$ and $d_{r}$, respectively. Radar working wavelength is $\lambda$.The signal emitts from the $m$-th antenna, is defined as $s_{m}(l)=\left[s_{m}(1), s_{m}(2), \cdots, s_{m}(L)\right]^{T}, m=1,2, \cdots, M$, where $L$ is the length of coded pulse signal, with $(\cdot)^{T}$ denoting the transpose of matrices. Assuming the different transmitting signals are orthogonal, then transmit steering vector of the array towards direction $\theta$ can be denoted as

$$
\boldsymbol{a}_{T}(\theta)=\left[1, e^{j 2 \pi \frac{d_{t} \cos \theta}{\lambda}}, \cdots, e^{j 2 \pi(M-1) \frac{d_{t} \cos \theta}{\lambda}}\right]^{T}
$$

and the receive steering vector is

$$
\boldsymbol{a}_{R}(\theta)=\left[1, e^{j 2 \pi \frac{d_{r} \cos \theta}{\lambda}}, \cdots, e^{j 2 \pi(N-1) \frac{d_{r} \cos \theta}{\lambda}}\right]^{T}
$$

Assuming that the target has a reflection coefficient $\beta(\theta)$, moves with an initial radial velocity $v$ and an acceleration $a$.The received signal at antenna $n$ and time index $l$ can be described by

$$
y_{n}(l)=\beta(\theta) e^{j 2 \pi(n-1) \frac{d_{r} \cos \theta}{\lambda}} \cdot e^{j 2 \pi f_{d} l} \cdot \sum_{m=1}^{M} s_{m}(l) e^{j 2 \pi(m-1) \frac{d_{t} \cos \theta}{\lambda}}+w_{n}(l)
$$


where $f_{d}$ represents Doppler shift caused by the maneuvering target, and $\boldsymbol{w}_{n}(l)$ is the noise components approximated by complex white Gaussian samples at the $n$-th receiver. From (3) the received space-time matrix can be expressed as

$$
\boldsymbol{y}(l)=\beta(\theta) \cdot \boldsymbol{a}_{R}(\theta) \boldsymbol{a}_{T}^{T}(\theta) \cdot s(l) \cdot e^{j 2 \pi f_{d} l}+\boldsymbol{w}(l)
$$

The total $L$ samples can be collected into a single matrix

$$
\boldsymbol{Y}=\beta(\theta) \cdot \boldsymbol{A}(\theta) \cdot \boldsymbol{S} \cdot \boldsymbol{D}\left(f_{d}\right)+\boldsymbol{W}
$$

where $\boldsymbol{A}(\theta)=\boldsymbol{a}_{R}(\theta) \boldsymbol{a}_{T}^{T}(\theta), \quad \boldsymbol{D}\left(f_{d}\right)=\operatorname{diag}\left(1, e^{j w_{t}}, \cdots, e^{j(L-1) w_{t}}\right) \quad$ is an $L \times L$ diagonal matrix, $\omega_{t}=2 \pi f_{d} / f_{r}=2 \pi \cdot 2 v \cdot T_{p} / \lambda+\pi \cdot 2 a \cdot T_{p}^{2} / \lambda$, where $T_{p}$ is the pulse width, and $W$ is an $N \times L$ noise matrix with a variance of $\sigma_{w}^{2}$.

\section{Maximum Likelihood Estimation}

Let $\psi=\left[\begin{array}{lll}\theta & \vee & a\end{array}\right]^{T}$ be the vector denoting the unknown parameters that need to be estimated. We use data $\boldsymbol{Y}$ as stated in (5) and be vectorized, then the received data vector $y$ can be obtained, which follows a Gaussian probability density function whose mean is $\gamma(\psi)=\operatorname{vec}\left(\beta(\theta) \boldsymbol{A}(\theta) \cdot \boldsymbol{S} \cdot \boldsymbol{D}\left(f_{d}\right)\right)$. Since a maneuvering target is discussed, the Doppler frequency $f_{d}$ involves two variables: initial velocity $v$ and acceleration $a$.

The probability density function of $y$ is

$$
\left.P\left(\boldsymbol{y} \mid \theta, f_{d}\right)=\frac{1}{\pi^{N L} \sigma^{2 N L}} e^{\left[-\frac{1}{\sigma_{w}^{2}}(y-\gamma(\psi))^{H}(y-\gamma(\psi))\right.}\right]
$$

where $(\cdot)^{H}$ denotes the conjugate transpose. The corresponding log-Likelihood function for radar data can then be described as

$$
P^{\prime}(\psi)=C-\frac{1}{\sigma_{w}^{2}}\left(\boldsymbol{y}^{H} \boldsymbol{y}-\boldsymbol{y}^{H} \boldsymbol{\gamma}\left(\theta, f_{d}\right)-\gamma\left(\theta, f_{d}\right)^{H} \boldsymbol{y}+\boldsymbol{\gamma}\left(\theta, f_{d}\right)^{H} \boldsymbol{\gamma}\left(\theta, f_{d}\right)\right)
$$

where $C$ is a constant which does not depend on $\psi$. To estimate the value of $\theta$ and $f_{d}$, the cost-function to be maximized is given by

$$
\begin{aligned}
\hat{\psi} & =\arg \max _{\psi}\left\{P^{\prime}(\psi)\right\} \\
& =\arg \min _{\psi}\left\{\boldsymbol{y}^{H} \boldsymbol{y}-\boldsymbol{y}^{H} \boldsymbol{\gamma}\left(\theta, f_{d}\right)-\boldsymbol{\gamma}\left(\theta, f_{d}\right)^{H} \boldsymbol{y}+\boldsymbol{\gamma}\left(\theta, f_{d}\right)^{H} \boldsymbol{\gamma}\left(\theta, f_{d}\right)\right\}
\end{aligned}
$$

The first term $y^{H} y$ in (8) does not depend on $\psi$. The final term $\gamma\left(\theta, f_{d}\right)^{H} \gamma\left(\theta, f_{d}\right)$ is constant with respect to $f_{d}$ and $\theta$. By exploiting the relation $\operatorname{vec}(\boldsymbol{A S D})=\left(\boldsymbol{D}^{T} \otimes \boldsymbol{A}\right)$ vec $(\boldsymbol{S})$, where $\otimes$ denotes Kronecker product, we have

$$
\begin{aligned}
\boldsymbol{\gamma}\left(\theta, f_{d}\right)^{H} \boldsymbol{\gamma}\left(\theta, f_{d}\right) & =\operatorname{vec}\left(\beta(\theta) \boldsymbol{A}(\theta) \boldsymbol{S} \boldsymbol{D}\left(f_{d}\right)\right)^{H} \operatorname{vec}\left(\beta(\theta) \boldsymbol{A}(\theta) \boldsymbol{S} \boldsymbol{D}\left(f_{d}\right)\right) \\
& =\left[\left(\boldsymbol{D}^{T}\left(f_{d}\right) \otimes \boldsymbol{A}(\theta)\right) \operatorname{vec}(\boldsymbol{S})\right]^{H}\left[\left(\boldsymbol{D}^{T}\left(f_{d}\right) \otimes \boldsymbol{A}(\theta)\right) \operatorname{vec}(\boldsymbol{S})\right] \\
& =\operatorname{vec}(\boldsymbol{S})^{H}\left(\boldsymbol{D}^{*}\left(f_{d}\right) \otimes \boldsymbol{A}^{H}(\theta)\right)\left(\boldsymbol{D}^{T}\left(f_{d}\right) \otimes \boldsymbol{A}(\theta)\right) \operatorname{vec}(\boldsymbol{S}) \\
& =\operatorname{vec}(\boldsymbol{S})^{H}\left[\left(\boldsymbol{D}^{*}\left(f_{d}\right) \boldsymbol{D}^{T}\left(f_{d}\right)\right) \otimes\left(\boldsymbol{A}^{H}(\theta) \boldsymbol{A}(\theta)\right)\right] \operatorname{vec}(\boldsymbol{S}) \\
& =\operatorname{vec}(\boldsymbol{S})^{H}\left[\boldsymbol{I}_{L \times L} \otimes\left(\boldsymbol{A}^{H}(\theta) \boldsymbol{A}(\theta)\right)\right] \operatorname{vec}(\boldsymbol{S}) \\
& =\left(N^{2} \times L\right) \cdot \boldsymbol{I}_{L \times L}
\end{aligned}
$$


It can be easily shown that the product in (9) yields a diagonal matrix with diagonal elements maintain a constant which equals to $N^{2} L$. Therefore the cost-function in (8) can be simplified as

$$
\hat{\psi}=\arg \max _{\psi}\left\{\boldsymbol{y}^{H} \gamma\left(\theta, f_{d}\right)+\gamma\left(\theta, f_{d}\right)^{H} \boldsymbol{y}\right\}
$$

In order to find the maximum likelihood estimations of the unknown parameters, it is necessary to perform a three-dimensional (3-D) search in the parameter space. From a practical point of view, such a 3-D search, for all possible values of DOA, velocity and acceleration of a target, can cause tremendous computational burden. Therefore, we propose a two-stage sequential estimation process.

As we simplify the target moving scenario by assuming the target is moving along a certain direction, a classical sub-space method, multiple signal classification, is used to estimate the DOA of the target which can be treated as static or relatively static. Then the estimation of initial velocity and acceleration can be obtained when the cost function in (10) achieves its maximum.

\section{Simulations}

We consider a MIMO radar with 6 antennas both on transmitting and receiving ends. Antennas are uniformly spaced with an distance of $\lambda / 2$, where $\lambda=0.23 \mathrm{~m}$. Each transmitter transmits different Hadamard codes with the same carrier frequency. The code length is set to $L=64$ and code duration is $T_{p}=5 \times 10^{-4} \mathrm{~s}$. The azimuth of the target is $\theta=45^{\circ}$, the initial velocity of the maneuvering target is $v=24.01 \mathrm{~m} / \mathrm{s}$ and acceleration is $a=99.9 \mathrm{~m} / \mathrm{s}^{2}$. Initial velocity searching range is from $0 \mathrm{~m} / \mathrm{s}$ to $40 \mathrm{~m} / \mathrm{s}$, acceleration searching range is from $70 \mathrm{~m} / \mathrm{s}^{2}$ to $130 \mathrm{~m} / \mathrm{s}^{2}$, the step for searching of initial velocity and acceleration are set to $0.25 \mathrm{~m} / \mathrm{s}$ and $0.05 \mathrm{~m} / \mathrm{s}^{2}$, respectively.

Monte Carlo simulations with 200 trials per signal-to-noise ratio (SNR) were conducted to acquire the RMSE of the ML estimation. The RMSE of DOA is shown in Figure 1, which indicates that MIMO radar has better angular estimation performance than phased array radar. The RMSE of initial velocity is shown in Figure 2, and that of acceleration is shown in Figure 3. Under various SNRs, MIMO radar and phased array radar both can obtain excellent estimation performance in estimating initial velocity and acceleration of maneuvering targets.

\section{Conclusions}

This paper studies the performance of parameters estimation of maneuvering target in coherent MIMO radars with the application of maximum likelihood estimation. Numerical simulation results show that MIMO radar has a better angular resolution than conventional phased array radar. The effectiveness of maximum likelihood estimator is also verified through the simulations.

\section{Acknowledgements}

This work is supported by National Nature Science Foundation of China (NSFC) 


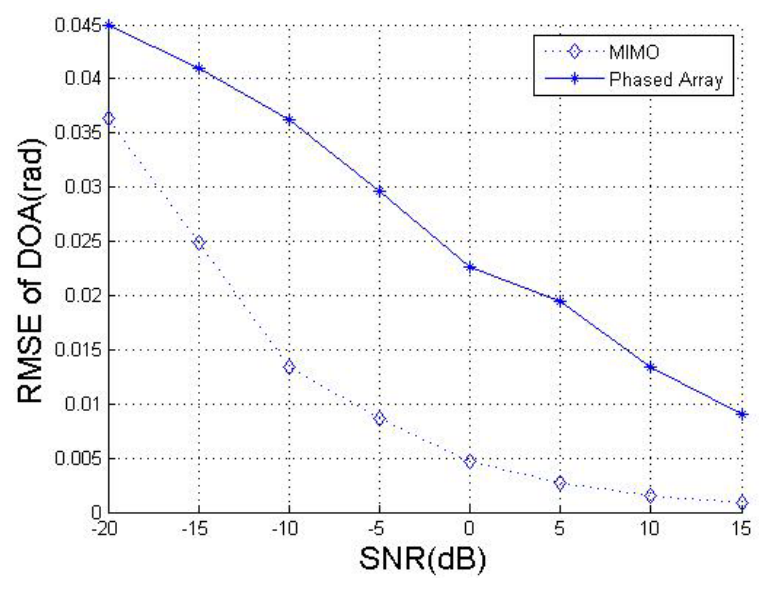

Figure 1. RMSE of DOA.

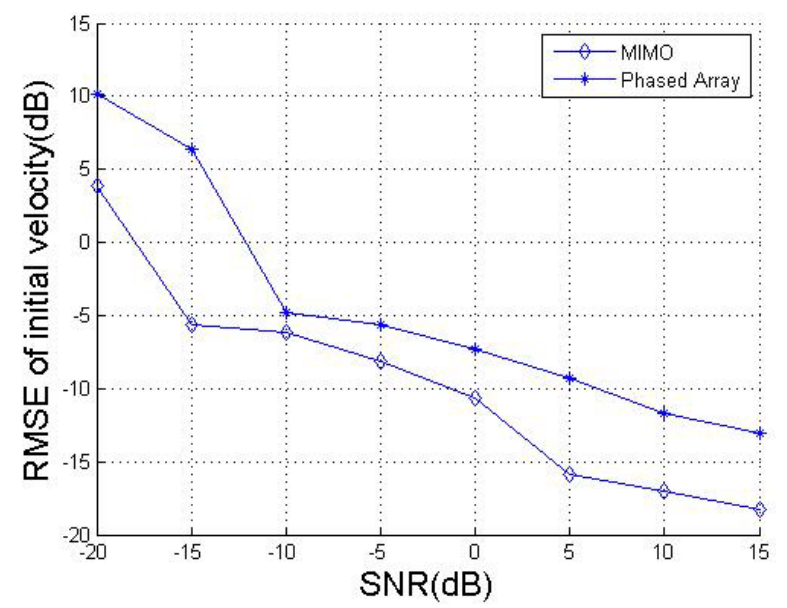

Figure 2. RMSE of initial velocity.

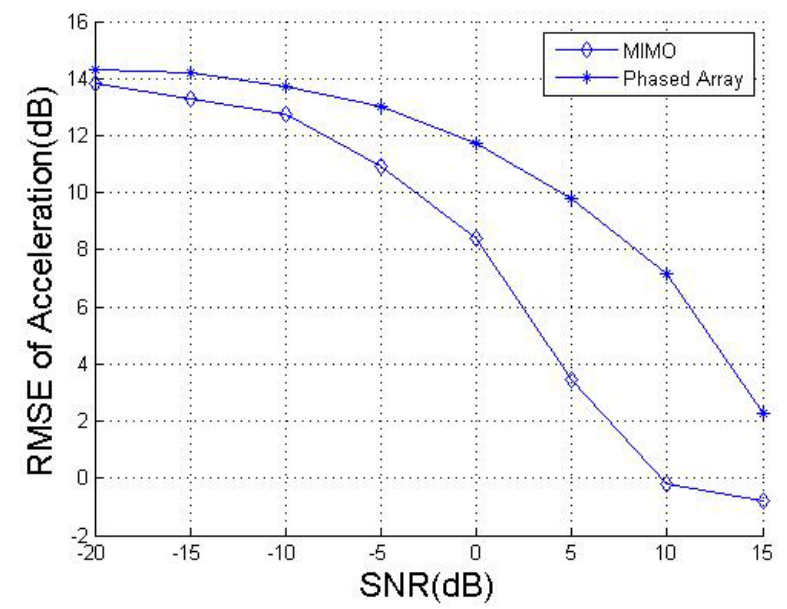

Figure 3. RMSE of acceleration.

under Grants 61471365, U1633107 and 61231017, National University's Basic Research Foundation of China under Grant No. 2000300446. The work is also supported by the Foundation for Sky Young Scholars of Civil Aviation University of China. 


\section{References}

[1] Goldsmith, A., Jafar, S.A., Jindal, N. and Vishwanath, S. (2003) Capacity Limits of MIMO Channels. IEEE Journal on Selected Areas in Communications, 21, 684-702. https://doi.org/10.1109/JSAC.2003.810294

[2] Cui, S.G., Goldsmith, A.J. and Bahai, A. (2004) Energy-Efficiency of MIMO and Cooperative MIMO Techniques in Sensor Networks. IEEE Journal on Selected Areas in Communications, 22, 1089-1098. https://doi.org/10.1109/JSAC.2004.830916

[3] Li, J. and Stoica, P. (2007) MIMO Radar with Colocated Antennas. IEEE Signal Processing Magazine, 24, 106-114. https://doi.org/10.1109/MSP.2007.904812

[4] Haimovich, A.M., Blum, R.S. and Cimini, L. (2008) MIMO Radar with Widely Separated Antennas. IEEE Signal Processing Magazine, 25, 116-129. https://doi.org/10.1109/MSP.2008.4408448

[5] Dang, X.F., Ma, K., Chen, B.X. and Yang, M.L. (2016) Direction of Arrival Estimation for Monostatic MIMO Radar Using Reduced-dimension RISR Algorithm. 11th International Symposium on Antennas, Propagation and EM Theory (ISAPE), Guilin, China, 18-21 October 2016, 607-611.

[6] Hokiguchi, M., Kikuma, N. and Sakakibara, K. (2016) Target Direction Estimation by MIMO Radar Using Root-MUSIC with Minimum Redundancy Array, International Symposium on Antennas and Propagation (ISAP), Okinawa, Japan, 24-28 October 2016, 1036-1037.

[7] He, Q., Blum, R.S., Godrich, H. and Haimovich, A.M. (2010) Target Velocity Estimation and Antenna Placement for MIMO Radar with Widely Separated Antennas. IEEE Journal of Selected Topics in Signal Processing, 4, 79-100. https://doi.org/10.1109/JSTSP.2009.2038974

[8] Jiang, M., Huang, J.G. and Hou, Y.S. (2010) MIMO Radar Joint Estimation of Target Location and Velocity with Multiple Subcarrier Signals. IEEE International Symposium on Phased Array Systems and Technology. Boston, 12-15 October 2010, 398-402. https://doi.org/10.1109/ARRAY.2010.5613338

[9] Sajjadieh, M.H.S. and Asif, A. (2015) Compressive Sensing Time Reversal MIMO Radar: Joint Direction and Doppler Frequency Estimation. IEEE Signal Processing Letters, 22, 1283-1287. https://doi.org/10.1109/LSP.2015.2396650

[10] Wang, N., Wang, W.G., Zhang, F. and Yuan, Y.N. (2014) The PARAFAC-MUSIC Algorithm for DOA Estimation with Doppler Frequency in a MIMO Radar System. International Journal of Antennas and Propagation, 2014, 1-5. https://doi.org/10.1155/2014/684591 
Submit or recommend next manuscript to SCIRP and we will provide best service for you:

Accepting pre-submission inquiries through Email, Facebook, LinkedIn, Twitter, etc. A wide selection of journals (inclusive of 9 subjects, more than 200 journals)

Providing 24-hour high-quality service

User-friendly online submission system

Fair and swift peer-review system

Efficient typesetting and proofreading procedure

Display of the result of downloads and visits, as well as the number of cited articles Maximum dissemination of your research work

Submit your manuscript at: http://papersubmission.scirp.org/

Or contact jec@scirp.org 